\title{
Precise intermittency for the parabolic Anderson equation with an $(1+1)$-dimensional time-space white noise
}

\author{
Xia Chen ${ }^{1}$ \\ Department of Mathematics, University of Tennessee, Knoxville, TN 37996, USA. E-mail: xchen@math.utk.edu
}

Received 13 December 2014; revised 23 February 2015; accepted 25 February 2015

\begin{abstract}
The moment Lyapunov exponent is computed for the solution of the parabolic Anderson equation with an $(1+1)$ dimensional time-space white noise. Our main result positively confirms an open problem posted in (Ann. Probab. (2015) to appear) and originated from the observations made in the physical literature (J. Statist. Phys. 78 (1995) 1377-1401) and (Nuclear Physics B 290 (1987) 582-602). By a link through the Feynman-Kac's formula, our theorem leads to the evaluation of the ground state energy for the $n$-body problem with Dirac pair interaction.
\end{abstract}

Résumé. Nous calculons les moments de l'exposant de Lyapunov de la solution de l'équation d'Anderson parabolique avec un bruit blanc en espace-temps en dimension $(1+1)$. Notre résultat principal confirme un problème ouvert posé dans (Ann. Probab. (2015) à paraître) et basé sur des observations faites dans la littérature physique (J. Statist. Phys. 78 (1995) 1377-1401) et (Nuclear Physics B 290 (1987) 582-602). À travers la formule de Feynman-Kac, notre théorème permet l'évaluation de l'état fondamental pour le problème à $n$-corps avec interaction de Dirac par paires.

MSC: 60F10; 60H15; 60H40; 60J65; $81 \mathrm{U} 10$

Keywords: Intermittency; White noise; Brownian motion; Parabolic Anderson model; Feynman-Kac's representation; Ground state energy

\section{Introduction}

We start with the $(1+1)$-dimensional parabolic Anderson equation

$$
\left\{\begin{array}{l}
\frac{\partial u}{\partial t}(t, x)=\frac{1}{2} \Delta u(t, x)+\lambda \dot{W}(t, x) u(t, x) \\
u(0, x)=u_{0}(x)
\end{array}\right.
$$

where $\dot{W}(t, x)\left((t, x) \in \mathbb{R}^{+} \times \mathbb{R}\right)$ is a time-space white noise defined as a centered generalized Gaussian field with the co-variance function

$$
\operatorname{Cov}(\dot{W}(s, x), \dot{W}(t, y))=\delta_{0}(s-t) \delta_{0}(x-y)(s, x), \quad(t, y) \in \mathbb{R}^{+} \times \mathbb{R}
$$

and $\lambda>0$ is a constant. Throughout the paper we assume in $(1.1)$ that $u_{0}(\cdot)$ is deterministic with

$$
0<\inf _{x \in \mathbb{R}} u_{0}(x) \leq \sup _{x \in \mathbb{R}} u_{0}(x)<\infty
$$

\footnotetext{
${ }^{1}$ Research partially supported by the Simons Foundation \#244767.
} 
The reader is referred to Dalang [9] and Walsh [18] for the mathematical set up of the system (1.1). This paper is mainly concerned about the evaluation of the moment Lyapunov exponent

$$
\lim _{t \rightarrow \infty} \frac{1}{t} \log \mathbb{E}\left(u(t, x)^{n}\right)
$$

for all integers $n \geq 1$. Since the Equation (1.1) is solved in the mild sense under the Dalang-Walsh's regime, $\mathbb{E} u(t, x)=\mathbb{E} u_{0}(x+B(t))$ where $B(t)$ is an 1-dimensional Brownian motion with $B(0)=0$. Consequently, the moment Lyapunov exponent is equal to 0 for $n=1$. So the problem remains only for $n \geq 2$.

A mathematical reduction by Feynman-Kac's formula (see Theorem 1.2 and its proof) relates this problem to the computation of

$$
\mathcal{E}_{n}(\theta)=\sup _{g \in \mathcal{F}_{n}}\left\{\theta \sum_{1 \leq j<k \leq n} \int_{\mathbb{R}^{n}} \delta_{0}\left(x_{j}-x_{k}\right) g^{2}(x) \mathrm{d} x-\frac{1}{2} \int_{\mathbb{R}^{n}}|\nabla g(x)|^{2} \mathrm{~d} x\right\}, \quad \theta>0,
$$

where we adopt the notation $x=\left(x_{1}, \ldots, x_{n}\right)$ and where $\mathcal{F}_{n}$ is the class of the infinitely smooth and rapidly decreasing (at infinity) functions $g$ on $\mathbb{R}^{n}$ with $\|g\|_{2}=1$. The variation $\mathcal{E}_{n}(\theta)$ appears as the ground state energy (or, the principal eigenvalue) of the $n$-body problem (see, e.g., [12] for the general background of the $n$-body problem) characterized by the Schrödinger Hamiltonian

$$
H_{n}=\frac{1}{2} \sum_{j=1}^{n} \Delta_{j}+\theta \sum_{1 \leq j<k \leq n} \delta_{0}\left(x_{j}-x_{k}\right) .
$$

Based on a physical method known as Bethe ansatz, Kardar ([14], Section 2.2) made a conjecture that

$$
\mathcal{E}_{n}(\theta)=\frac{\theta^{2}}{24} n\left(n^{2}-1\right), \quad n=2,3, \ldots
$$

by proposing the ground state wave function (principal eigenfunction) as

$$
g_{0}(x)=\exp \left\{-\theta \sum_{1 \leq j<k \leq n}\left|x_{j}-x_{k}\right|\right\}
$$

where $x=\left(x_{1}, \ldots, x_{n}\right) \in \mathbb{R}^{n}$. With the convention that $\frac{\mathrm{d}}{\mathrm{d} z}|z|=\operatorname{sgn}(z)$ and $\frac{\mathrm{d}^{2}}{\mathrm{~d} z^{2}}|z|=2 \delta_{0}(z)(z \in \mathbb{R})$ and with a formal computation, Kardar is able to claim that

$$
\left(H_{n} g_{0}\right)(x)=\frac{\theta^{2}}{24} n\left(n^{2}-1\right) g_{0}(x) .
$$

In addition to the non-smoothness in the super planes $x_{j}=x_{k}(1 \leq j<k \leq n)$, a worse problem of $g_{0}(\cdot)$ is that it does not vanish at infinity and does not belong to $\mathcal{L}^{2}\left(\mathbb{R}^{n}\right)$. Nevertheless, Kardar's expository argument provides insight as what to expect.

An attempt for rigorous treatment was made by Bertini and Cancrini [2] who claimed the Feynman-Kac type moment formula

$$
\mathbb{E} u(t, x)^{n}=2 \exp \left\{t \lambda^{4} \frac{n\left(n^{2}-1\right)}{24}\right\} \Phi\left(t \lambda^{2}\left(\frac{n\left(n^{2}-1\right)}{12}\right)^{1 / 2}\right)
$$

under the uniform initial condition $u_{0}(x)=1$, where $\Phi(\cdot)$ is the distribution function for 1-dimensional standard normal distribution. Unfortunately, Equation (1.5) is correct only for $n=2-$ the error in Bertini-Cancrini's computation comes from an incorrect use of Skorokhod's lemma. To disprove (1.5), notice that (see (3.1) with $u_{0}(x)=1$ for the left-hand side) the left and right-hand sides are the generating functions (in $\lambda^{2}$ ) of the random variables

$$
\sum_{1 \leq j<k \leq n} \int_{0}^{t} \delta_{0}\left(B_{j}(s)-B_{k}(s)\right) \mathrm{d} s \text { and }\left(\frac{n\left(n^{2}-1\right)}{12}\right)^{1 / 2}|B(t)|,
$$


respectively, where $B(t)$ is an 1-dimensional Brownian motion with $B(0)=0$. On the other hand, these two random variables are distributed differently when $n \geq 3$, simply because they have different expectations

$$
\frac{n(n-1)}{2 \sqrt{\pi}} t \text { and }\left(\frac{n\left(n^{2}-1\right)}{6 \pi}\right)^{1 / 2} t .
$$

To compute the expectations of the local times appearing on the left-hand side, here we have used the fact that $B(s) \equiv\left(B_{j}(s)-B_{k}(s)\right) / \sqrt{2}$ is a Brownian motion so

$$
\int_{0}^{t} \delta_{0}\left(B_{j}(s)-B_{k}(s)\right) \mathrm{d} s=\frac{1}{\sqrt{2}} \int_{0}^{t} \delta_{0}(B(s)) \mathrm{d} s \stackrel{d}{=} \frac{1}{\sqrt{2}}|B(t)|,
$$

where " $\stackrel{d}{=}$ " follows the well-known identity in law between the Brownian local time and the reflected Brownian motion.

Despite of the mistake, some later development in this area suggests that the Bertini-Cancrini's idea is asymptotically close to the truth. The author ([6], Proposition 3.3, see also Remark 3.1 below for a short alternative proof) recently pointed out that

$$
\lim _{n \rightarrow \infty} n^{-3} \log \mathbb{E} u(t, x)^{n}=\frac{1}{24} \lambda^{4}, \quad \forall t>0, x \in \mathbb{R}
$$

which shows that the Bertini-Cancrini's idea can be approached by the high moment asymptotics.

Recently, Joseph, Khoshnevisan and Mueller [13] post an open problem conjecturing that

$$
\lim _{t \rightarrow \infty} \frac{1}{t} \log \mathbb{E} u(t, x)^{n}=\frac{1}{24} n\left(n^{2}-1\right) \lambda^{4}
$$

for all integers $n=2,3, \ldots$. If confirmed, it indicates that the Bertini-Cancrini's idea can also be approximated by the long-term asymptotics. Finally, Bertini and Cancrini [2] deserve the credit for their idea of using Tanaka's formula in the moment computation for (1.1) which plays an essential role in our paper.

The parabolic Anderson equation given in (1.1) has become increasingly important partially due to its connection the Kardar-Parisi-Zhang's equation (KPZ equation) (see [15] and [16] for the physical background)

$$
\frac{\partial h}{\partial t}(t, x)=\frac{1}{2} \frac{\partial^{2} h}{\partial x^{2}}(t, x)+\frac{1}{2}\left(\frac{\partial h}{\partial x}(t, x)\right)^{2}+\lambda \dot{W}(t, x), \quad(t, x) \in \mathbb{R}^{+} \times \mathbb{R}
$$

through the Hopf-Cole's transform $u(t, x)=\exp \{h(t, x)\}$ (here we take risk of over-simplification - see [10] for detail), and to the monumental work by Hairer [10] in the study of the KPZ equation. In the wake of the historic breakthrough of the KPZ equation, it is the time to set the record straight by giving a rigorous proof of (1.4) and by answering the open problem (1.7).

Theorem 1.1. (1.7) holds for every $n=2,3, \ldots$, and $x \in \mathbb{R}$.

In their celebrated paper [1], Amir, Corwin and Quastel remark ([1], p. 472) that the main physical prediction on the long term asymptotic laws for the KPZ equation is based on the exact computation of moment Lyaponov exponent for the parabolic Anderson equation (1.1). To see how the physical prediction is made, we start with the problem of the quenched Lyapunov exponent. From his physical derivation of (1.4), Kardar ([14], Section 3.3) infers that a version of (1.7) holds even for the fractional $n$, especially for the small $n>0$ and carries out the following heuristic computation

$$
\begin{aligned}
\lim _{t \rightarrow \infty} \frac{1}{t} \mathbb{E} \log u(t, x) & =\lim _{t \rightarrow \infty} \frac{1}{t} \lim _{n \rightarrow 0^{+}} \frac{1}{n}\left\{\mathbb{E} u(t, x)^{n}-1\right\} \\
& =\lim _{t \rightarrow \infty} \lim _{n \rightarrow 0^{+}} \frac{1}{n t}\left(\exp \left\{n t\left(n^{2}-1\right) \frac{\lambda^{4}}{24}\right\}-1\right)=-\frac{\lambda^{4}}{24} .
\end{aligned}
$$


Kardar's prediction about the above limit turns out to be right, as later proved by Bertini and Giacomin [3] who state that under the initial condition $u_{0}(x)=\exp \{w(x)\}$ with $w(x)(x \in \mathbb{R})$ being a bilateral Brownian motion,

$$
\lim _{t \rightarrow \infty} \frac{1}{t} \int_{\mathbb{R}^{d}} \varphi(x) \log u(t, x) \mathrm{d} x=-\frac{\lambda^{4}}{24} \int_{\mathbb{R}} \varphi(x) \mathrm{d} x \quad \text { in } L^{2}(\Omega, \mathcal{A}, \mathbb{P})
$$

for every $\varphi \in C_{0}^{\infty}(\mathbb{R})$. More than what is stated in (1.9), Bertini and Giacomin [3] actually establish the second moment bound

$$
\mathbb{E}\left\{\int_{\mathbb{R}^{d}} \varphi(x) \log u(t, x) \mathrm{d} x+\frac{\lambda^{4} t}{24} \int_{\mathbb{R}} \varphi(x) \mathrm{d} x\right\}^{2} \leq C t^{2 / 3}
$$

which was later developed by Amir, Gorwin and Quastel ([1], Corollary 1.3) into a law of the second order claiming that for any fixed $x \in \mathbb{R}$, under the initial condition $u_{0}(\cdot)=\delta_{x}(\cdot)$ the process

$$
t^{-1 / 3}\left\{\log u(t, x)+\frac{\lambda^{4} t}{24}\right\}
$$

weakly converges, as $t \rightarrow \infty$, to the GUE Tracy-Widom distribution, i.e., the limiting distribution of the scaled and centered largest eigenvalue of the Gaussian unitary random matrices (the coefficient $\lambda=1$ in [1] - we put $\lambda$ here for the purpose of comparison). Under the Hopf-Cole's transform $u(t, x)=\exp \{h(t, x)\}$, it leads to the second order fluctuation law for the KPZ equation (1.8) [1].

Here we try to learn from Kardar's view for making further predictions. We begin with the simple observation that (1.7) can be re-written as

$$
\lim _{t \rightarrow \infty} \frac{1}{t} \log \mathbb{E} \exp \left\{n\left(\log u(t, x)+\frac{\lambda^{4} t}{24}\right)\right\}=\frac{\lambda^{4} n^{3}}{24}, \quad n=1,2, \ldots
$$

A natural question is whether or not this remains true when $n$ is replaced by any non-negative number. An even more exciting question is what if $n$ is replaced by a positive and deterministic function $\varphi(t)$ that goes to zero with a proper rate as $t \rightarrow \infty$. In particular, we replace $n$ by

$$
\varphi(t)=\theta\left(\frac{l(t)}{t}\right)^{1 / 3}
$$

where $\theta>0$ is an arbitrary constant and $l(t) \uparrow \infty(t \rightarrow \infty)$ is slowly varying. In view of (1.11), we have reason to believe that

$$
\lim _{t \rightarrow \infty} \frac{1}{l(t)} \log \mathbb{E} \exp \left\{\theta\left(\frac{l(t)}{t}\right)^{1 / 3}\left(\log u(t, x)+\frac{\lambda^{4} t}{24}\right)\right\}=\frac{\lambda^{4} \theta^{3}}{24}, \quad \theta>0 .
$$

In light of Gärtner-Ellis theorem (see, e.g., [5], Theorem 1.2.4, p. 11), we expect that

$$
\lim _{t \rightarrow \infty} \frac{1}{l(t)} \log \mathbb{P}\left\{\log u(t, x)+\frac{\lambda^{4} t}{24} \geq a t^{1 / 3} l(t)^{2 / 3}\right\}=-\sup _{\theta>0}\left\{a \theta-\frac{\lambda^{4} \theta^{3}}{24}\right\}=-\frac{4 \sqrt{2}}{3 \lambda^{2}} a^{3 / 2}
$$

for any $a>0$. The likelihood of such result is indicated by the weak convergence obtained by Amir, Gorwin and Quastel ([1], Corollary 1.3). We leave it to future investigation.

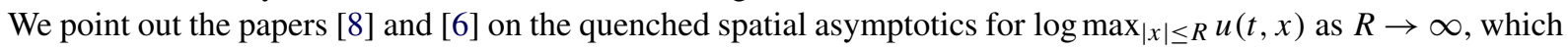
is substantially relevant to the high moment asymptotics given in (1.6).

Finally, we refer the interested reader to [7], Theorem 6.1, for the precise moment Lyapunov exponents in the setting of fractional noise and of weak solution.

The limit given in (1.7), denoted by $\gamma_{n}$, satisfies $\gamma_{n} / n<\gamma_{n+1} /(n+1)(n=1,2, \ldots)$, the relation that defines intermittency. It suggests that in the long run, the space is dominated by the areas where $u(t, x)$ is low with a few exceptional "isolated island" where $u(t, x)$ achieves high peaks. See [4] for details about the notion of intermittency. 
Theorem 1.2. For every $n=2,3, \ldots$ and $\theta>0(1.4)$ holds.

\section{An inequality in number theory}

Let $b_{1}, \ldots, b_{n}$ be distinct real numbers and $\mathbf{1}_{i, j}(1 \leq i, j \leq n$ and $i \neq j)$ be numbers which are equal to 0 or 1 and assume that $\mathbf{1}_{i, j}=\mathbf{1}_{j, i}$ for all $1 \leq i, j \leq n$ with $i \neq j$. Our goal in this section is to prove the following proposition.

\section{Proposition 2.1.}

$$
\begin{aligned}
& \sum_{j=1}^{n}\left(\sum_{i \neq j} \operatorname{sgn}\left(b_{i}-b_{j}\right)\right)^{2}=\frac{1}{3} n\left(n^{2}-1\right), \\
& \sum_{j=1}^{n}\left(\sum_{i \neq j} \operatorname{sgn}\left(b_{i}-b_{j}\right) \mathbf{1}_{i, j}\right)^{2} \leq \frac{1}{3} n\left(n^{2}-1\right) .
\end{aligned}
$$

The equality (2.1) has been noted in Kardar [14] and Bertini-Cancrini [2]. We include it here for the reader's convenience. By permutation invariance

$$
\sum_{j=1}^{n}\left(\sum_{i \neq j} \operatorname{sgn}\left(b_{i}-b_{j}\right)\right)^{2}=\sum_{j=1}^{n}\left(\sum_{i \neq j} \operatorname{sgn}\left(b_{\sigma(i)}-b_{\sigma(j)}\right)\right)^{2}
$$

for any permutation $\sigma$ on $\{1, \ldots, n\}$. Here we pick $\sigma$ to make $b_{\sigma(1)}>\cdots>b_{\sigma(n)}$. Consequently,

$$
\sum_{j=1}^{n}\left(\sum_{i \neq j} \operatorname{sgn}\left(b_{i}-b_{j}\right)\right)^{2}=\sum_{j=1}^{n}((n-1)-2(j-1))^{2}=\frac{n\left(n^{2}-1\right)}{3} .
$$

Inequality (2.2) appears to be new. Its proof is much harder than expected. The idea is to transform the problem into some matrix computations. Given an $n \times n$ matrix $A=\left(a_{i, j}\right)$, write $s_{j}(A)$ as the sum of the entries in the $j$ th column of $A$. Set

$$
\beta(A)=\sum_{j=1}^{n}\left(\sum_{i=1}^{n} a_{i, j}\right)^{2}=\sum_{j=1}^{n} s_{j}(A)^{2}
$$

For any $1 \leq j<i \leq n$, let $C(i, j)$ denote the $n \times n$ skew-symmetric matrix with the $(i, j)$-entry equal to -1 , the $(j, i)$-entry equal to 1 and other entries equal to 0 .

Lemma 2.2. Let $1 \leq j<i \leq n$. For any $(n \times n)$ skew-symmetric matrices $M$ and $N$ satisfying $M=N+C(i, j)$ and $s_{i}(M)-s_{j}(M) \geq 1, \beta(M) \geq \beta(N)$.

Proof. Clearly, $s_{i}(C(i, j))=1$ and $s_{j}(C(i, j))=-1$. Hence,

$$
s_{i}(M)=s_{i}(N)+s_{i}(C(i, j))=s_{i}(N)+1, \quad s_{j}(M)=s_{j}(N)+s_{j}(C(i, j))=s_{i}(N)-1 .
$$

Thus, $s_{i}(M)-s_{j}(M)=s_{i}(N)-s_{j}(N)+2$.

Let $S(N C(i, j))$ be the sum of all entries of the $(n \times n)$-matrix $N C(i, j)$. Notice that the $j$ th column of the $n \times n$ matrix $N C(i, j)$ is the negative of the $i$ th column of $N$, and the $i$ th column of $N C(i, j)$ is the $j$ th column of $N$. The other columns of $N C(i, j)$ are 0 . Consequently,

$$
S(N C(i, j))=-s_{i}(N)+s_{j}(N)=2-\left(s_{i}(M)-s_{j}(M)\right) .
$$


Let $\alpha$ be the $(n \times 1)$-matrix with all entries equal to 1 . We have that

$$
\alpha^{T} N C(i, j) \alpha=S(N C(i, j))=2-\left(s_{i}(M)-s_{j}(M)\right) .
$$

In addition, $\beta(M)=\left\|\alpha^{T} M\right\|^{2}=\left\|\alpha^{T}(N+C(i, j))\right\|^{2}, \beta(N)=\left\|\alpha^{T} N\right\|^{2}$ and $\left\|\alpha^{T} C(i, j)\right\|^{2}=2$. By the skewsymmetry of $C(i, j)$, therefore,

$$
\begin{aligned}
\beta(M) & =\beta(N)+2+2 \alpha^{T} N C(i, j)^{T} \alpha=\beta(N)+2-2 \alpha^{T} N C(i, j) \alpha \\
& =\beta(N)+2\left(s_{i}(M)-s_{j}(M)\right)-2 \geq \beta(N) .
\end{aligned}
$$

Proof of (2.2). By permutation invariance, we may assume that $b_{1}>b_{2}>\cdots>b_{n}$. So the $(n \times n)$-matrix $M_{0}=$ $\left(\operatorname{sgn}\left(b_{i}-b_{j}\right)\right)$ becomes a skew-symmetric matrix with the entries in the upper-triangle equal to 1 , the entries in the lower-triangle equal to -1 , and the entries on the diagonal equal to zero (for this we need the agreement that $\operatorname{sgn}(0)=0)$. According to $(2.1)$,

$$
\beta\left(M_{0}\right)=\frac{1}{3} n\left(n^{2}-1\right) .
$$

Let $\left(i_{1}, j_{1}\right), \ldots,\left(i_{k}, j_{k}\right)$ be the zeros of $\mathbf{1}_{i, j}$ with $i_{1}>j_{1}, \ldots, i_{k}>j_{k}$ and set

$$
M_{k}=M_{0}-\sum_{l=1}^{k} C\left(i_{l}, j_{l}\right) .
$$

A crucial observation is that $M_{k}$ can be obtained by killing all lower triangle entries with labels $\left(i_{1}, j_{1}\right), \ldots,\left(i_{k}, j_{k}\right)$ and their upper-triangle reflections in $M_{0}$, i.e., replacing them by 0 . Therefore, all we need is to show that $\beta\left(M_{0}\right) \geq \beta\left(M_{k}\right)$. To apply Lemma 2.2, we proceed in $k$ steps, killing one symmetric pair at a time.

A subtle issue is the order of our steps. In order to validate the condition $s_{i}(\cdot)-s_{j}(\cdot) \geq 1$ posted in Lemma 2.2, we re-label the entries $\left(i_{1}, j_{1}\right), \ldots,\left(i_{k}, j_{k}\right)$ so that $i_{1}-j_{1} \leq \cdots \leq i_{k}-j_{k}$. Then we define

$$
M_{l}=M_{l-1}-C\left(i_{l}, j_{l}\right), \quad l=1, \ldots, k .
$$

Clearly, the definition of $M_{k}$ here is consistent with how $M_{k}$ was defined before the re-labeling. We now show that under this arrangement, one can do even better than what is required by Lemma 2.2. In fact, we claim that

$$
s_{i_{l}}\left(M_{l-1}\right)-s_{j_{l}}\left(M_{l-1}\right) \geq 2, \quad l=1, \ldots, k .
$$

Let $1 \leq l \leq k$ be fixed and notice that $M_{l-1}$ is transformed from $M_{0}$ by killing the symmetric pairs

$$
\left\{\left(i_{l}, j_{1}\right),\left(j_{1}, i_{1}\right)\right\}, \ldots,\left\{\left(i_{l-1}, j_{l-1}\right),\left(j_{l-1}, i_{l-1}\right)\right\}
$$

one pair at a time, in $l-1$ steps. Before the steps are taken,

$$
s_{i_{l}}\left(M_{0}\right)-s_{j_{l}}\left(M_{0}\right)=2\left(i_{l}-j_{l}\right) .
$$

Under our arrangement, there is no simultaneous killing of column- $i_{l}$ and column- $j_{l}$ entries in the first $l-1$ steps. Hence, each step results in exactly one of the three consequences:

(1) A killing occurs in the column $i_{i}$.

(2) A killing occurs in the column $j_{l}$.

(3) There is no killing in the $i_{l}$ th column and in the $j_{l}$ th column.

In any one of the first $l-1$ steps, the worst thing is that the quantity

$$
s_{i_{l}}(\cdot)-s_{j_{l}}(\cdot)
$$


decreases by 1 (otherwise the quantity either remains unchanged or increases by 1 ). It happens only when a “ +1 "-entry in the $i_{l}$-column is killed, or when a "- 1 "-entry in the $j_{l}$-column is killed. Therefore,

$$
s_{i_{l}}\left(M_{l-1}\right)-s_{j_{l}}\left(M_{l-1}\right) \geq 2\left(i_{l}-j_{l}\right)-H_{l-1}^{+}-H_{l-1}^{-},
$$

where $H_{l-1}^{+}$and $H_{l-1}^{-}$are, respectively, the number of the positive killings in the $i_{l}$ th column and the number of the negative killings in the $j_{l}$ th column among the first $l-1$ steps. Since the positive entries are located in the upper triangle region and negative entries are located in the lower triangle region, the positive killings can only happen at the locations $\left(j_{1}, i_{1}\right), \ldots,\left(j_{l-1}, i_{l-1}\right)$ and the negative killings can only happen at the locations $\left(i_{1}, j_{1}\right), \ldots,\left(i_{l-1}, j_{l-1}\right)$. Hence,

$$
H_{l-1}^{+}=\#\left\{1 \leq b \leq l-1 ; i_{b}=i_{l}\right\} \quad \text { and } \quad H_{l-1}^{-}=\#\left\{1 \leq b \leq l-1 ; j_{b}=j_{l}\right\} .
$$

For any $1 \leq b \leq l-1$ with $i_{b}=i_{l}$, we have that $j_{b}<i_{b}=i_{l}$ and $j_{b} \neq j_{l}$. Further, $i_{l}-j_{b}=i_{b}-j_{b} \leq i_{l}-j_{l}$ or, $j_{b} \geq j_{l}$. In summary, we have that $j_{b} \in\left[j_{l}+1, i_{l}-1\right]$ if $i_{l}-j_{l} \geq 2$ (so the integer interval $\left[j_{l}+1, i_{l}-1\right] \neq \varnothing$ ). The same argument also shows that $\left\{1 \leq b \leq l-1 ; i_{b}=i_{l}\right\}=\varnothing$ when $i_{l}-j_{l}=1$. In the case when $i_{l}-j_{l} \geq 2$, for any distinct $1 \leq b, b^{\prime} \leq l-1$ with $i_{b}=i_{b^{\prime}}=i_{l}$, we have that $j_{b} \neq j_{b^{\prime}}$. Consequently, $H_{l-1}^{+} \leq i_{l}-j_{l}-1$ regardless $i_{l}-j_{l}=1$ or $i_{l}-j_{l} \geq 2$. A similar argument gives that $H_{l-1}^{-} \leq i_{l}-j_{l}-1$. Thus,

$$
s_{i_{l}}\left(M_{l-1}\right)-s_{j_{l}}\left(M_{l-1}\right) \geq 2\left(i_{l}-j_{l}\right)-\left(i_{l}-j_{l}-1\right)-\left(i_{l}-j_{l}-1\right) \geq 2 .
$$

So we have (2.5).

Applying Lemma 2.2 to $M=M_{l-1}$ and $N=M_{l}$ we have $\beta\left(M_{l-1}\right) \geq \beta\left(M_{l}\right)$ for $l=1, \ldots, k$. This leads to $\beta\left(M_{0}\right) \geq \beta\left(M_{k}\right)$.

For possible future reference, we remark that the inequality in (2.2) can be sharpened to the following form:

$$
\sum_{j=1}^{n}\left(\sum_{i \neq j} \operatorname{sgn}\left(b_{i}-b_{j}\right) \mathbf{1}_{i, j}\right)^{2} \leq \frac{1}{3} n\left(n^{2}-1\right)-2 \cdot \#\left\{(i, j) ; 1 \leq j<i \leq n, \mathbf{1}_{i, j}=0\right\} .
$$

Indeed, (2.7) follows from an obvious modification of (2.4) which gives $\beta\left(M_{l-1}\right) \geq \beta\left(M_{l}\right)+2$ for $l=1, \ldots, k$ under (2.5). On the other hand, (2.7) cannot be strengthened into equality. Take $n=3$ and $b_{1}>b_{2}>b_{3}, 1_{1,3}=1_{3,1}=0$ and $1_{i, j}=1$ for other $(i, j)$. Then the left-hand side of (2.7) is 2 while the right-hand side is 6 .

\section{Proof of Theorem 1.1}

By the moment representation ([11], Theorem 5.3),

$$
\mathbb{E} u(t, x)^{n}=\mathbb{E}\left(\exp \left\{\lambda^{2} \sum_{1 \leq j<k \leq n} \int_{0}^{t} \delta_{0}\left(B_{j}(s)-B_{k}(s)\right) \mathrm{d} s\right\} \prod_{j=1}^{n} u_{0}\left(x+B_{j}(t)\right)\right),
$$

where $B_{1}(s), \ldots, B_{n}(s)$ are i.i.d. 1-dimensional Brownian motions starting at 0 and the time integrals represent the Brownian local times. In view of the assumption (1.2), all we need is to show that for any $\theta>0$

$$
\lim _{t \rightarrow \infty} \frac{1}{t} \log \mathbb{E} \exp \left\{\theta \sum_{1 \leq j<k \leq n} \int_{0}^{t} \delta_{0}\left(B_{j}(s)-B_{k}(s)\right) \mathrm{d} s\right\}=\frac{\theta^{2}}{24} n\left(n^{2}-1\right) .
$$

The lower bound for (3.2) essentially follows from the strategy developed by Bertini and Cancrini [2], which is included here for the reader's convenience. Applying Tanaka's formula ([17], Theorem 1.2, p. 207) to the Brownian 
motion $\left(B_{j}(s)-B_{k}(s)\right) / \sqrt{2}$ gives

$$
\begin{aligned}
& \left|\frac{B_{j}(t)-B_{k}(t)}{\sqrt{2}}\right| \\
& \quad=\frac{1}{\sqrt{2}} \int_{0}^{t} \operatorname{sgn}\left(B_{j}(s)-B_{k}(s)\right) \mathrm{d}\left(B_{j}(s)-B_{k}(s)\right)+\int_{0}^{t} \delta_{0}\left(\frac{B_{j}(s)-B_{k}(s)}{\sqrt{2}}\right) \mathrm{d} s \\
& \quad=\frac{1}{\sqrt{2}} \int_{0}^{t} \operatorname{sgn}\left(B_{j}(s)-B_{k}(s)\right) \mathrm{d}\left(B_{j}(s)-B_{k}(s)\right)+\sqrt{2} \int_{0}^{t} \delta_{0}\left(B_{j}(s)-B_{k}(s)\right) \mathrm{d} s .
\end{aligned}
$$

Consequently,

$$
\int_{0}^{t} \delta_{0}\left(B_{j}(s)-B_{k}(s)\right) \mathrm{d} s \geq \frac{1}{2} \int_{0}^{t} \operatorname{sgn}\left(B_{k}(s)-B_{j}(s)\right) \mathrm{d}\left(B_{j}(s)-B_{k}(s)\right)
$$

and therefore,

$$
\begin{aligned}
& \sum_{1 \leq j<k \leq n} \int_{0}^{t} \delta_{0}\left(B_{j}(s)-B_{k}(s)\right) \mathrm{d} s \\
& \geq \frac{1}{2} \sum_{1 \leq j<k \leq n} \int_{0}^{t} \operatorname{sgn}\left(B_{k}(s)-B_{j}(s)\right) \mathrm{d}\left(B_{j}(s)-B_{k}(s)\right) \\
& =\frac{1}{2} \sum_{j=1}^{n} \int_{0}^{t}\left(\sum_{k \neq j} \operatorname{sgn}\left(B_{k}(s)-B_{j}(s)\right)\right) \mathrm{d} B_{j}(s) .
\end{aligned}
$$

As a process in $t$, the $n$-summation on the right-hand side is a continuous martingale. By (2.1), it has a deterministic quadratic variation

$$
\int_{0}^{t} \sum_{j=1}^{n}\left(\sum_{k \neq j} \operatorname{sgn}\left(B_{k}(s)-B_{j}(s)\right)\right)^{2} \mathrm{~d} s=t \frac{n\left(n^{2}-1\right)}{3} .
$$

Consequently ([17], Theorem 1.6, p. 170),

$$
\sum_{j=1}^{n} \int_{0}^{t}\left(\sum_{k \neq j} \operatorname{sgn}\left(B_{k}(s)-B_{j}(s)\right)\right) \mathrm{d} B_{j}(s) \stackrel{d}{=} B\left(t \frac{n\left(n^{2}-1\right)}{3}\right),
$$

where $B(t)$ is an 1-dimensional Brownian motion with $B(0)=0$.

Therefore,

$$
\begin{aligned}
& \mathbb{E} \exp \left\{\theta \sum_{1 \leq j<k \leq n} \int_{0}^{t} \delta_{0}\left(B_{j}(s)-B_{k}(s)\right) \mathrm{d} s\right\} \\
& \geq \mathbb{E} \exp \left\{\frac{\theta}{2} B\left(t \frac{n\left(n^{2}-1\right)}{3}\right)\right\}=\exp \left\{\frac{\theta^{2}}{8} \frac{n\left(n^{2}-1\right)}{3} t\right\} .
\end{aligned}
$$

This leads to the lower bound

$$
\liminf _{t \rightarrow \infty} \frac{1}{t} \log \mathbb{E} \exp \left\{\theta \sum_{1 \leq j<k \leq n} \int_{0}^{t} \delta_{0}\left(B_{j}(s)-B_{k}(s)\right) \mathrm{d} s\right\} \geq \frac{\theta^{2}}{24} n\left(n^{2}-1\right) .
$$


We now consider the upper bound. Applying Skorokhod's lemma ([17], Lemma 2.1, p. 222) to Equation (3.3) with

$$
z(t)=\left|B_{j}(t)-B_{k}(t)\right| \text { and } a(t)=2 \int_{0}^{t} \delta_{0}\left(B_{j}(s)-B_{k}(s)\right) \mathrm{d} s
$$

gives that for any $1 \leq j<k \leq n$

$$
\int_{0}^{t} \delta_{0}\left(B_{j}(s)-B_{k}(s)\right) \mathrm{d} s=\frac{1}{2} \sup _{s \leq t} \int_{0}^{s} \operatorname{sgn}\left(B_{k}(u)-B_{j}(u)\right) \mathrm{d}\left(B_{j}(u)-B_{k}(u)\right) .
$$

Set

$$
B_{j, k}(t)=\frac{1}{\sqrt{2}} \int_{0}^{t} \operatorname{sgn}\left(B_{k}(u)-B_{j}(u)\right) \mathrm{d}\left(B_{j}(u)-B_{k}(u)\right) .
$$

Consequently,

$$
\begin{aligned}
\sum_{1 \leq j<k \leq n} \int_{0}^{t} \delta_{0}\left(B_{j}(s)-B_{k}(s)\right) \mathrm{d} s & =\frac{1}{\sqrt{2}} \sum_{1 \leq j<k \leq n} \sup _{s \leq t} B_{j, k}(s) \\
& =\frac{1}{\sqrt{2}} \sup _{\mathbf{s} \in[0, t]^{\mathcal{A}}} \sum_{1 \leq j<k \leq n} B_{j, k}\left(s_{j, k}\right)=\frac{1}{\sqrt{2}} \sup _{\mathbf{s} \in[0, t]^{\mathcal{A}}} G(\mathbf{s}),
\end{aligned}
$$

where $G(\mathbf{s})$ is a multi-parameter process given as

$$
G(\mathbf{s})=\sum_{1 \leq j<k \leq n} B_{j, k}\left(s_{j, k}\right), \quad \mathbf{s}=\left(s_{j, k} ; 1 \leq j<k \leq n\right) \in\left(\mathbb{R}^{+}\right)^{\mathcal{A}}
$$

and we adopt the notations

$$
\mathcal{A}=\{(j, k) ; 1 \leq j<k \leq n\}, \quad \mathbf{s}=\left(s_{j, k} ; 1 \leq j<k \leq n\right) .
$$

Notice that the quadratic variation of $B_{j, k}(t)$ is

$$
\int_{0}^{t}\left[\operatorname{sgn}\left(B_{k}(s)-B_{j}(s)\right)\right]^{2} \mathrm{~d} s=t .
$$

Consequently ([17], Theorem 1.6, p. 170), $B_{j, k}(t)$ is a Brownian motion. In spite of that, we are not able to show that $\left\{G(\mathbf{s}) ; \mathbf{s} \in[0, t]^{\mathcal{A}}\right\}$ is a Gaussian field. Our strategy is parameter discretization. Let the integer $m \geq 1$ be large but fixed and let $0=t_{0}<t_{1}<\cdots<t_{m}=t$ be a uniform partition. Set $\Pi_{m}=\{1, \ldots, m\}^{\mathcal{A}}$ and partition $[0, t]^{\mathcal{A}}$ into

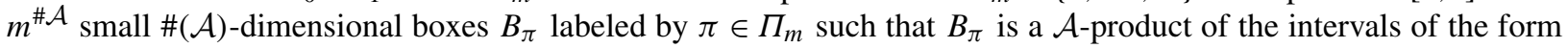
$\left[t_{i-1}, t_{i}\right](i=1, \ldots, m)$. By $(3.6)$

$$
\begin{aligned}
\mathbb{E} & \exp \left\{\theta \sum_{1 \leq j<k \leq n} \int_{0}^{t} \delta_{0}\left(B_{j}(s)-B_{k}(s)\right) \mathrm{d} s\right\} \\
& =\mathbb{E} \exp \left\{\frac{\theta}{\sqrt{2}} \max _{\pi \in \Pi_{m}} \sup _{\mathbf{s} \in B_{\pi}} G(\mathbf{s})\right\} \\
& \leq \sum_{\pi \in \Pi_{m}} \mathbb{E} \exp \left\{\frac{\theta}{\sqrt{2}} \sup _{\mathbf{s} \in B_{\pi}} G(\mathbf{s})\right\} .
\end{aligned}
$$

Let the box $B_{\pi}$ be fixed and write

$$
B_{\pi}=\prod_{(j, k) \in \mathcal{A}}\left[s_{j, k}, t_{j, k}\right]=\left[\mathbf{s}_{\pi}, \mathbf{t}_{\pi}\right]
$$


By Hölder's inequality,

$$
\begin{aligned}
\mathbb{E} & \exp \left\{\frac{\theta}{\sqrt{2}} \sup _{\mathbf{s} \in B_{\pi}} G(\mathbf{s})\right\} \\
& \leq\left(\mathbb{E} \exp \left\{\frac{p \theta}{\sqrt{2}} G\left(\mathbf{s}_{\pi}\right)\right\}\right)^{1 / p}\left(\mathbb{E} \exp \left\{\frac{q \theta}{\sqrt{2}} \sup _{\mathbf{s} \in B_{\pi}}\left(G(\mathbf{s})-G\left(\mathbf{s}_{\pi}\right)\right)\right\}\right)^{1 / q},
\end{aligned}
$$

where $p, q>1$ are fixed conjugate numbers with $p$ close to 1 . Using Hölder's inequality again,

$$
\begin{aligned}
& \mathbb{E} \exp \left\{\frac{q \theta}{\sqrt{2}} \sup _{\mathbf{s} \in B_{\pi}}\left(G(\mathbf{s})-G\left(\mathbf{s}_{\pi}\right)\right)\right\} \\
& \leq \prod_{(j, k) \in \mathcal{A}}\left(\mathbb{E} \exp \left\{\frac{q n(n-1) \theta}{2 \sqrt{2}} \sup _{s \in\left[s_{j, k}, t_{j, k}\right]}\left(B_{j, k}(s)-B_{j, k}\left(s_{j, k}\right)\right)\right\}\right)^{2 /(n(n-1))} \\
& =\mathbb{E} \exp \left\{\frac{q n(n-1) \theta}{2 \sqrt{2}} \sqrt{\frac{t}{m}}|B(1)|\right\} \leq 2 \exp \left\{\frac{1}{16}(q n(n-1) \theta)^{2} \frac{t}{m}\right\} .
\end{aligned}
$$

In addition, notice that

$$
\begin{aligned}
G\left(\mathbf{s}_{\pi}\right) & =\frac{1}{\sqrt{2}} \sum_{1 \leq j<k \leq n} \int_{0}^{s_{j, k}} \operatorname{sgn}\left(B_{k}(u)-B_{j}(u)\right) \mathrm{d}\left(B_{j}(u)-B_{k}(u)\right) \\
& =\frac{1}{\sqrt{2}} \sum_{j=1}^{n} \sum_{k \neq j} \int_{0}^{s_{j, k}} \operatorname{sgn}\left(B_{k}(u)-B_{j}(u)\right) \mathrm{d} B_{j}(u) \\
& =\frac{1}{\sqrt{2}} \sum_{j=1}^{n} \sum_{k \neq j} \int_{0}^{t} \operatorname{sgn}\left(B_{k}(u)-B_{j}(u)\right) 1_{\left[0, s_{j, k}\right]}(u) \mathrm{d} B_{j}(u) \\
& =\frac{1}{\sqrt{2}} \sum_{j=1}^{n} \int_{0}^{t}\left(\sum_{k \neq j} \operatorname{sgn}\left(B_{k}(u)-B_{j}(u)\right) 1_{\left[0, s_{j, k}\right]}(u)\right) \mathrm{d} B_{j}(u) .
\end{aligned}
$$

Here we extend the definition of $s_{j, k}$ as $s_{j, k}=s_{k, j}$ for $j>k$.

By Ito's formula,

$$
\mathbb{E} \exp \left\{\frac{p \theta}{\sqrt{2}} G\left(\mathbf{s}_{\pi}\right)-\frac{(p \theta)^{2}}{8} \sum_{j=1}^{n} \int_{0}^{t}\left(\sum_{k \neq j} \operatorname{sgn}\left(B_{k}(u)-B_{j}(u)\right) 1_{\left[0, s_{j, k}\right]}(u)\right)^{2} \mathrm{~d} u\right\}=1 .
$$

By (2.2), for each $0 \leq u \leq t$

$$
\sum_{j=1}^{n}\left(\sum_{k \neq j} \operatorname{sgn}\left(B_{k}(u)-B_{j}(u)\right) 1_{\left[0, s_{j, k}\right]}(u)\right)^{2} \leq \frac{1}{3} n\left(n^{2}-1\right) .
$$

Consequently,

$$
\sum_{j=1}^{n} \int_{0}^{t}\left(\sum_{k \neq j} \operatorname{sgn}\left(B_{k}(u)-B_{j}(u)\right) 1_{\left[0, s_{j, k}\right]}(u)\right)^{2} \mathrm{~d} u \leq t \frac{n\left(n^{2}-1\right)}{3} .
$$

Thus,

$$
\mathbb{E} \exp \left\{\frac{p \theta}{\sqrt{2}} G\left(\mathbf{s}_{\pi}\right)\right\} \leq \exp \left\{\frac{(p \theta)^{2}}{24} n\left(n^{2}-1\right) t\right\} .
$$


Summarizing our steps since (3.7),

$$
\begin{aligned}
& \mathbb{E} \exp \left\{\theta \sum_{1 \leq j<k \leq n} \int_{0}^{t} \delta_{0}\left(B_{j}(s)-B_{k}(s)\right) \mathrm{d} s\right\} \\
& \leq 2 m^{(n(n-1)) / 2} \exp \left\{\frac{1}{16} q(n(n-1) \theta)^{2} \frac{t}{m}\right\} \exp \left\{\frac{p \theta^{2}}{24} n\left(n^{2}-1\right) t\right\} .
\end{aligned}
$$

So we have

$$
\begin{aligned}
& \limsup _{t \rightarrow \infty} \frac{1}{t} \log \mathbb{E} \exp \left\{\theta \sum_{1 \leq j<k \leq n} \int_{0}^{t} \delta_{0}\left(B_{j}(s)-B_{k}(s)\right) \mathrm{d} s\right\} \\
& \leq \frac{q(n(n-1) \theta)^{2}}{16 m}+\frac{p \theta^{2}}{24} n\left(n^{2}-1\right) .
\end{aligned}
$$

Letting $m \rightarrow \infty$, and then $p \rightarrow 1^{+}$on the right-hand side leads to the upper bound

$$
\limsup _{t \rightarrow \infty} \frac{1}{t} \log \mathbb{E} \exp \left\{\theta \sum_{1 \leq j<k \leq n} \int_{0}^{t} \delta_{0}\left(B_{j}(s)-B_{k}(s)\right) \mathrm{d} s\right\} \leq \frac{\theta^{2}}{24} n\left(n^{2}-1\right) .
$$

Finally, (3.2) follows from (3.5) and (3.9).

Remark 3.1. A slight modification of the above argument also leads to an alternative proof of (1.6), which is equivalent to

$$
\lim _{n \rightarrow \infty} n^{-3} \log \mathbb{E} \exp \left\{\theta \sum_{1 \leq j<k \leq n} \int_{0}^{t} \delta_{0}\left(B_{j}(s)-B_{k}(s)\right) \mathrm{d} s\right\}=\frac{\theta^{2}}{24} t \quad(\theta, t>0)
$$

under the initial condition (1.2). Indeed, the lower bound for (3.10) follows from (3.4). As for the upper bound, the only thing we need to revise in the approximation (3.8) is to allow $m$ to grow with $n$ in a proper rate. An easy choice is to let $m=n^{2}$.

\section{Proof of Theorem 1.2}

In view of (3.2), all we need is to show that

$$
\lim _{t \rightarrow \infty} \frac{1}{t} \log \mathbb{E} \exp \left\{\theta \sum_{1 \leq j<k \leq n} \int_{0}^{t} \delta_{0}\left(B_{j}(s)-B_{k}(s)\right) \mathrm{d} s\right\}=\mathcal{E}_{n}(\theta) .
$$

This claim brings no surprise as it is a typical consequence of the Feynman-Kac's theory on the Markovian semigroup. The only technical obstacle is the singularity of Dirac function. For this reason we first replace $\delta_{0}(\cdot)$ by a nice function. More specifically, by Theorem 4.1.6, p. 99, [5]

$$
\begin{aligned}
& \lim _{t \rightarrow \infty} \frac{1}{t} \log \mathbb{E} \exp \left\{\theta \sum_{1 \leq j<k \leq n} \int_{0}^{t} p_{\varepsilon}\left(B_{j}(s)-B_{k}(s)\right) \mathrm{d} s\right\} \\
& =\sup _{g \in \mathcal{F}_{n}}\left\{\theta \sum_{1 \leq j<k \leq n} \int_{\mathbb{R}^{n}} p_{\varepsilon}\left(x_{j}-x_{k}\right) g^{2}(x) \mathrm{d} x-\frac{1}{2} \int_{\mathbb{R}^{n}}|\nabla g(x)|^{2} \mathrm{~d} x\right\}
\end{aligned}
$$

for every $\varepsilon>0$, where $p_{\varepsilon}(\cdot)$ is the density of $B(\varepsilon)$, and $x=\left(x_{1}, \ldots, x_{n}\right) \in \mathbb{R}^{d}$. 
By Fourier inversion

$$
\begin{aligned}
& \sum_{1 \leq j<k \leq n} \int_{0}^{t} p_{\varepsilon}\left(B_{j}(s)-B_{k}(s)\right) \mathrm{d} s \\
& =\frac{1}{2 \pi} \int_{-\infty}^{\infty} \exp \left\{-\frac{\varepsilon}{2}|\xi|^{2}\right\}\left[\int_{0}^{t}\left(\sum_{1 \leq j<k \leq n} \exp \left\{\mathrm{i} \xi\left(B_{k}(s)-B_{j}(s)\right)\right\}\right) \mathrm{d} s\right] \mathrm{d} \xi
\end{aligned}
$$

Hence, for any integer $m \geq 1$,

$$
\begin{aligned}
& \mathbb{E}\left[\sum_{1 \leq j<k \leq n} \int_{0}^{t} p_{\varepsilon}\left(B_{j}(s)-B_{k}(s)\right) \mathrm{d} s\right]^{m} \\
&=\frac{1}{(2 \pi)^{m}} \int_{\mathbb{R}^{m}} \int_{[0, t]^{m}} \mathrm{~d} s_{1} \xi_{1} \cdots \mathrm{d} s_{m} \mathrm{~d} \xi_{m} \exp \left\{-\frac{\varepsilon}{2} \sum_{l=1}^{m}\left|\xi_{l}\right|^{2}\right\} \\
& \\
& \quad \times\left(\mathbb{E} \prod_{l=1}^{m} \sum_{1 \leq j<k \leq n} \exp \left\{\mathrm{i} \xi_{l}\left(B_{k}(s)-B_{j}(s)\right)\right\}\right) \\
& \leq \frac{1}{(2 \pi)^{m}} \int_{\mathbb{R}^{m}} \int_{[0, t]^{m}} \mathrm{~d} s_{1} \xi_{1} \cdots \mathrm{d} s_{m} \mathrm{~d} \xi_{m}\left(\mathbb{E} \prod_{l=1}^{m} \sum_{1 \leq j<k \leq n} \exp \left\{\mathrm{i} \xi_{l}\left(B_{k}(s)-B_{j}(s)\right)\right\}\right) \\
&= \mathbb{E}\left[\sum_{1 \leq j<k \leq n} \int_{0}^{t} \delta_{0}\left(B_{j}(s)-B_{k}(s)\right) \mathrm{d} s\right]^{m},
\end{aligned}
$$

where the inequality follows from the fact that

$$
\mathbb{E} \prod_{l=1}^{m} \sum_{1 \leq j<k \leq n} \exp \left\{\mathrm{i} \xi_{l}\left(B_{k}(s)-B_{j}(s)\right)\right\}>0 .
$$

By Taylor's expansion, we have that

$$
\begin{aligned}
& \mathbb{E} \exp \left\{\theta \sum_{1 \leq j<k \leq n} \int_{0}^{t} p_{\varepsilon}\left(B_{j}(s)-B_{k}(s)\right) \mathrm{d} s\right\} \\
& \leq \mathbb{E} \exp \left\{\theta \sum_{1 \leq j<k \leq n} \int_{0}^{t} \delta_{0}\left(B_{j}(s)-B_{k}(s)\right) \mathrm{d} s\right\} .
\end{aligned}
$$

In view of (4.2), therefore,

$$
\begin{aligned}
& \liminf _{t \rightarrow \infty} \frac{1}{t} \log \mathbb{E} \exp \left\{\theta \sum_{1 \leq j<k \leq n} \int_{0}^{t} \delta_{0}\left(B_{j}(s)-B_{k}(s)\right) \mathrm{d} s\right\} \\
& \geq \sup _{g \in \mathcal{F}_{n}}\left\{\theta \sum_{1 \leq j<k \leq n} \int_{\mathbb{R}^{n}} p_{\varepsilon}\left(x_{j}-x_{k}\right) g^{2}(x) \mathrm{d} x-\frac{1}{2} \int_{\mathbb{R}^{n}}|\nabla g(x)|^{2} \mathrm{~d} x\right\} .
\end{aligned}
$$

Letting $\varepsilon \rightarrow 0^{+}$on the right-hand side leads to the lower bound

$$
\liminf _{t \rightarrow \infty} \frac{1}{t} \log \mathbb{E} \exp \left\{\theta \sum_{1 \leq j<k \leq n} \int_{0}^{t} \delta_{0}\left(B_{j}(s)-B_{k}(s)\right) \mathrm{d} s\right\} \geq \mathcal{E}_{n}(\theta) .
$$


To establish the upper bound we first claim that for any $\theta>0$

$$
\underset{\varepsilon \rightarrow 0^{+}}{\limsup \limsup } \frac{1}{t \rightarrow \infty} \frac{1}{t} \log \mathbb{E} \exp \left\{\theta \sum_{1 \leq j<k \leq n} \int_{0}^{t}\left(\delta_{0}-p_{\varepsilon}\right)\left(B_{j}(s)-B_{k}(s)\right) \mathrm{d} s\right\} \leq 0 .
$$

Indeed, for any $t_{1}, t_{2}>0$, by Markov property,

$$
\begin{aligned}
& \mathbb{E} \exp \left\{\theta \sum_{1 \leq j<k \leq n} \int_{0}^{t_{1}+t_{2}}\left(\delta_{0}-p_{\varepsilon}\right)\left(B_{j}(s)-B_{k}(s)\right) \mathrm{d} s\right\} \\
& \leq \mathbb{E} \exp \left\{\theta \sum_{1 \leq j<k \leq n} \int_{0}^{t_{1}}\left(\delta_{0}-p_{\varepsilon}\right)\left(B_{j}(s)-B_{k}(s)\right) \mathrm{d} s\right\} \\
& \quad \times \sup _{x_{j, k}} \mathbb{E} \exp \left\{\theta \sum_{1 \leq j<k \leq n} \int_{0}^{t_{2}}\left(\delta_{0}-p_{\varepsilon}\right)\left(x_{j, k}+B_{j}(s)-B_{k}(s)\right) \mathrm{d} s\right\} .
\end{aligned}
$$

Similar as (4.3), one can prove that

$$
\begin{gathered}
\mathbb{E} \exp \left\{\theta \sum_{1 \leq j<k \leq n} \int_{0}^{t_{2}}\left(\delta_{0}-p_{\varepsilon}\right)\left(x_{j, k}+B_{j}(s)-B_{k}(s)\right) \mathrm{d} s\right\} \\
\leq \mathbb{E} \exp \left\{\theta \sum_{1 \leq j<k \leq n} \int_{0}^{t_{2}}\left(\delta_{0}-p_{\varepsilon}\right)\left(B_{j}(s)-B_{k}(s)\right) \mathrm{d} s\right\}
\end{gathered}
$$

for any $x_{j, k} \in \mathbb{R}(1 \leq j, k \leq n)$. Therefore, we conclude that

$$
\begin{aligned}
& \mathbb{E} \exp \left\{\theta \sum_{1 \leq j<k \leq n} \int_{0}^{t_{1}+t_{2}}\left(\delta_{0}-p_{\varepsilon}\right)\left(B_{j}(s)-B_{k}(s)\right) \mathrm{d} s\right\} \\
& \leq \mathbb{E} \exp \left\{\theta \sum_{1 \leq j<k \leq n} \int_{0}^{t_{1}}\left(\delta_{0}-p_{\varepsilon}\right)\left(B_{j}(s)-B_{k}(s)\right) \mathrm{d} s\right\} \\
& \times \mathbb{E} \exp \left\{\theta \sum_{1 \leq j<k \leq n} \int_{0}^{t_{2}}\left(\delta_{0}-p_{\varepsilon}\right)\left(B_{j}(s)-B_{k}(s)\right) \mathrm{d} s\right\} .
\end{aligned}
$$

This leads to the following inequality:

$$
\begin{aligned}
& \mathbb{E} \exp \left\{\theta \sum_{1 \leq j<k \leq n} \int_{0}^{t}\left(\delta_{0}-p_{\varepsilon}\right)\left(B_{j}(s)-B_{k}(s)\right) \mathrm{d} s\right\} \\
& \leq\left(\mathbb{E} \exp \left\{\theta \sum_{1 \leq j<k \leq n} \int_{0}^{1}\left(\delta_{0}-p_{\varepsilon}\right)\left(B_{j}(s)-B_{k}(s)\right) \mathrm{d} s\right\}\right)^{t}
\end{aligned}
$$

for any $t>1$. Consequently, the claim (4.5) follows from the obvious fact that

$$
\lim _{\varepsilon \rightarrow 0^{+}} \mathbb{E} \exp \left\{\theta \sum_{1 \leq j<k \leq n} \int_{0}^{1}\left(\delta_{0}-p_{\varepsilon}\right)\left(B_{j}(s)-B_{k}(s)\right) \mathrm{d} s\right\}=1 .
$$

Finally, the requested upper bound follows from (4.2), (4.5) and a standard procedure of the exponential approximation by Hölder's inequality. 


\section{Acknowledgements}

The author would like to thank his colleague Dr. Shashikant Mulay for his help in proving the inequality (2.2). Gratitude also goes to the anonymous referee for detecting a gap in the proof of the inequality (2.2) and for making numerous corrections and suggestions.

\section{References}

[1] G. Amir, I. Corwin and J. Quastel. Probability distribution of the free energy of the continuum directed random polymer in $1+1$ dimensions. Comm. Pure Appl. Math. 64 (2011) 466-537. MR2796514

[2] L. Bertini and N. Cancrini. The stochastic heat equation: Feynman-Kac formula and intermittence. J. Stat. Phys. 78 (1995) $1377-1401$. MR1316109

[3] L. Bertini and G. Giacomin. On the long time behavior of the stochastic heat equation. Probab. Theory Related Fields 114 (1999) $279-289$. MR1705123

[4] R. A. Carmona and S. A. Molchanov. Parabolic Anderson model and intermittency. Mem. Amer. Math. Soc. 108 (1994) 1-125. MR1185878

[5] X. Chen. Random Walk Intersections: Large Deviations and Related Topics. Mathematical Surveys and Monographs 157. Amer. Math. Soc., Providence, RI, 2009. MR2584458

[6] X. Chen. Spatial asymptotics for the parabolic Anderson models with generalized time-space Gaussian noise. Ann. Probab. To appear, 2015.

[7] X. Chen, Y. Z. Hu, J. Song and F. Xing. Exponential asymptotics for time-space Hamiltonians. Ann. Inst. Henri Poincaré. To appear, 2015.

[8] D. Conus, M. Joseph and D. Khoshnevisan. On the chaotic character of the stochastic heat equation, before the onset of intermittency. Ann. Probab. 41 (2013) 2225-2260. MR3098071

[9] R. C. Dalang. Extending martingale measure stochastic integral with applications to spatially homogeneous S.P.D.E's. Electron. J. Probab. 4 (1999) 1-29. MR1684157

[10] M. Hairer. Solving the KPZ equation. Ann. of Math. (2) 178 (2013) 559-664. MR3071506

[11] Y. Z. Hu and D. Nualart. Stochastic heat equation driven by fractional noise and local time. Probab. Theory Related Fields 143 (2009) 285-328. MR2449130

[12] W. Hunziker and I. M. Sigal. The quantum N-body problem. J. Math. Phys. 41 (2000) 3448-3510. MR1768629

[13] M. Joseph, D. Khoshnevisan and C. Mueller. Strong invariance and noise-comparison principle for some parabolic stochastic PDEs. Ann. Probab. To appear, 2015.

[14] M. Kardar. Replica Bethe ansatz studies of two-dimensional interfaces with quenched random impurities. Nuclear Phys. B 290 (1987) 582602. MR0922846

[15] M. Kardar, G. Parisi and Y. C. Zhang. Dynamic scaling of growing interface. Phys. Rev. Lett. 56 (1986) 889-892.

[16] M. Kardar and Y. C. Zhang. Scaling of directed polymers in random media. Phys. Rev. Lett. 58 (1987) 2087-2090.

[17] D. Revuz and M. Yor. Continuous Martingale and Brownian Motion, 2nd edition. Springer, Berlin, 1994. MR1303781

[18] J. B. Walsh. An introduction to stochastic partial differential equations. In École d'Été de Probabilités de Saint-Flour XIV-1984 $265-439$. Lecture Notes in Math. 1180. Springer, Berlin, 1986. MR0876085 